Bibliotecas. Vol 35, № 1, enero-junio, 2017, pp.1-19. EISSN: 1659-3286

URL: http://www.revistas.una.ac.cr/index.php/bibliotecas/index

DOI: $\underline{\text { http://dx.doi.org/10.15359/rb.35-1.2 }}$

Licencia: Creative Commons (BY-NC-ND) 4.0 Internacional

\title{
Normas básicas de seguridad y salud para edificios de biblioteca según las Normas Mexicanas (NOM)
}

\section{Basic Safety and Health Standards for Buildings of Library According to the Mexican standards}

\author{
César S. Olguín Camacho * \\ Biblioteca del Programa Universitario de Estudios sobre la Ciudad, \\ UNAM, México \\ Elsa Galicia Hernández ** \\ Dirección General de Bibliotecas, UNAM, México
}

Recibido: 01 de marzo de 2016 Aceptado: 15 de noviembre del 2016

Corregido: 23 de enero de 2017 Publicado: 05 de mayo de 2017

\begin{abstract}
Resumen
Las bibliotecas están expuestas a cierto tipo de destrucción que va desde modificaciones leves hasta graves, producto de factores como el fuego, el agua, derrumbes y temblores. Es importante mencionar que si se cuenta con los equipos necesarios y las precauciones que eviten estos riesgos, el daño será menor.

Se requiere entonces conocer las condiciones establecidas sobre seguridad y salud por la normatividad mexicana sobre espacios e instalaciones de índole laboral que son aplicables por tanto a bibliotecas. Por lo tanto se mencionarán los puntos que permitan vislumbrar los requerimientos básicos que ayuden a las bibliotecas a tener, de manera clara, las normas sobre seguridad y salud que aseguren la estancia segura del personal y de los usuarios.
\end{abstract}

Palabras clave: Seguridad, Higiene, Salud, Bibliotecas y Normas mexicanas.

\begin{abstract}
The libraries are exposed to a certain type of destruction that ranges from mild to severe and the main factors involved are fire, water, landslides and earthquakes, it is important to mention that if you have the necessary equipment and precautions to avoid these risks, the damage will be less.

It is important to know the conditions established safety and health by Mexican standards on space and facilities for labor issues, which are applicable for both libraries.

So the points that allow a glimpse of the basic requirements that help libraries have concisely the safety and health standards that ensure the stay of staff and users will be mentioned.
\end{abstract}

Keywords: Safety, Hygiene, Health, Libraries and mexican Standards 


\section{Introducción}

El tema de las bibliotecas abarca infinidad de aspectos, pero el tema de seguridad, higiene y salud en relación con las bibliotecas en México no ha sido tan bordado. Cuando se construyen nuevos edificios para bibliotecas o espacios adaptados debe tomarse en cuenta las normas básicas para cumplir con los requisitos básicos de comodidad, estética y aprovechamiento de los espacios. Las autoridades mexicanas competentes de establecer la normatividad a los espacios, expone aquellos puntos respecto a la seguridad y salud que atañe a los edificios de las bibliotecas.

Como espacio de trabajo y por la cantidad de flujo de personas que se transitan en las bibliotecas es necesario aplicar la normatividad que aminore los riesgos, accidentes y daños al acervo de la biblioteca así como al bibliotecario y los usuarios; por lo tanto, es importante conocer qué dicen las normas oficiales mexicanas y qué tanto se conocen y cumplen dentro de los espacios o edificios de las bibliotecas, sin importar el tamaño.

\section{a. Contexto nacional}

México ha legislado desde 1917 la materia laboral, teniendo como objetivo mejorar las condiciones de vida y trabajo de los empleados. Esto implica la mejora de las condiciones de los lugares de trabajo, áreas y edificios que garanticen las medidas adecuadas para la prevención de accidentes de las personas que trabajen (bibliotecarios) o asistan (usuarios) a ellos.

Actualmente se cuenta con diversas instancias que regulan y marcan los diferentes preceptos de la seguridad e higiene en México. Se cuenta con la Ley Federal de Trabajo de 2015, la Ley Federal sobre Metrología Normalización 2015, el Reglamento Federal de Seguridad y Salud en el Trabajo 2014, todas ellas normas generales, pero que pueden adaptarse en nuestro caso a las bibliotecas, acervos, bibliotecarios y usuarios.

La Ley Federal del Trabajo en su artículo 132, fracción XVI y XVII menciona:

$\mathrm{XVI}$. Instalar y operar las fábricas, talleres, oficinas, locales y demás lugares en que deban ejecutarse las labores, de acuerdo con las disposiciones establecidas en el reglamento y las 
normas oficiales mexicanas en materia de seguridad, salud y medio ambiente de trabajo, a efecto de prevenir accidentes y enfermedades laborales. Asimismo, deberán adoptar las medidas preventivas y correctivas que determine la autoridad laboral;

XVII. Cumplir el reglamento y las normas oficiales mexicanas en materia de seguridad, salud y medio ambiente de trabajo, así como disponer en todo tiempo de los medicamentos y materiales de curación indispensables para prestar oportuna y eficazmente los primeros auxilios.

La Secretaría del Trabajo y Previsión Social (STPS) es la instancia facultada para estudiar y ordenar las medidas de seguridad e higiene industriales para la protección de los trabajadores. Emite las Normas Oficiales Mexicanas (NOM) que determinan las condiciones mínimas necesarias para la prevención de riesgos de trabajo. Actualmente hay 41 normas en materia de seguridad y salud en el trabajo, las cuales se agrupan en cinco categorías: de seguridad, salud, organización, específicas y de producto.

Para el análisis de este trabajo se toman como base las normas de seguridad y salud, actualmente compuestas por 18 normas, de estas se tomaron cuatro acordes con los edificios o espacios para bibliotecas:

- NOM-001-STPS-2008. Edificios, locales e instalaciones (Seguridad)

- NOM-002-STPS-2010. Prevención protección contra incendios (Seguridad)

- NOM-011-STPS-2011. Ruido, y

- NOM-025-STPS-2008. Iluminación (Salud).

\section{b. Biblioteca}

La biblioteca es un espacio dedicado tanto al estudio como al espaciamiento. Su objetivo es proporcionar diversos servicios a la comunidad, para ello debe contar con espacios acordes al número de usuarios que atiende, cumplir con colores, espacios, señalización para un mejor desenvolvimiento de las personas dentro de las instalaciones. Como organismo de servicios responsable de salvaguardar la información y como espacio para el estudio y la investigación, debe ofrecer a su personal y usuarios un espacio para el desarrollo de sus labores de trabajo con seguridad. Para ello debe cubrir los requerimientos de seguridad e 
higiene establecidos por la STPS, independientemente del tipo de biblioteca que sea, el acervo que contenga o su tamaño.

El objetivo de presentar estos puntos es promover la importancia de la seguridad e higiene y su cumplimento basado en normas nacionales, con sustento en la reglamentación vigente en la materia. Además se quiere impulsar la mejora continua en la prevención de accidentes y enfermedades de trabajo con el fin de disminuirlos o evitarlos en las bibliotecas.

Los espacios de una biblioteca deben someterse a la protección de las instalaciones físicas, deterioros o destrucciones de acervos mobiliarios, herramientas, materiales y equipo, así como ser parte de una política de previsión y protección contra incendios y condiciones ambientales. La higiene y la seguridad son parte esencial del quehacer dentro de las bibliotecas y comprende procedimientos, técnicas y elementos para el reconocimiento, evaluación y control de los agentes nocivos que intervienen en los procesos y actividades de trabajo, a fin de conservar la vida, salud e integridad física de los trabajadores y evitar cualquier posible deterioro al propio centro de trabajo. (Reglamento de Seguridad, Higiene y Medio ambiente 1997). ${ }^{1}$

Los artículos e incisos de la normativa que se describen a continuación se presentarán tal y como aparecen en las normas, adecuándose a espacios o bibliotecas para alterar la idea original de la norma a la biblioteca. El campo de aplicación de las Normas se rige en todo el territorio nacional y aplica para todos los centros de trabajo, por ende, es posible aplicarla a las bibliotecas.

\section{Norma Oficial Mexicana nom-002-STPS-2010, Edificios, locales, instalaciones y áreas en los centros de trabajo-condiciones de seguridad}

Esta norma tiene por objetivo establecer las condiciones de seguridad, locales e instalaciones y áreas en los centros de trabajo para su adecuado funcionamiento y conservación, con la finalidad de prevenir riesgos a los bibliotecarios como trabajadores. 


\section{a. Obligaciones del patrón ${ }^{2}$}

- Conservar en condiciones seguras las instalaciones de las bibliotecas (centros de trabajo), para que no representen riesgos.

- Realizar verificaciones oculares cada doce meses al centro de trabajo, pudiendo hacerse por áreas, para identificar condiciones inseguras y reparar los daños encontrados.

- Efectuar verificaciones oculares posteriores a la ocurrencia de un evento que pudiera generarle daños al centro de trabajo y, en su caso, realizar las adecuaciones, modificaciones o reparaciones que garanticen la seguridad de sus ocupantes.

- Contar con sanitarios (retretes, mingitorios, lavabos, entre otros) limpios y seguros para el servicio de los trabajadores y, en su caso, con lugares reservados para el consumo de alimentos.

\section{b.Obligaciones de los trabajadores (bibliotecarios)}

- Informar al patrón las condiciones inseguras que detecten en el centro de trabajo.

- Participar en la conservación del centro de trabajo y dar a las áreas el uso para el que fueron destinadas, a menos que el patrón autorice su empleo para otros usos.

\section{c. Requisitos de seguridad en el centro de trabajo (bibliotecas)}

- Contar con orden y limpieza permanentes en las áreas de trabajo, así como en pasillos exteriores a los edificios, estacionamientos y otras áreas comunes del centro de trabajo, de acuerdo con el tipo de actividades que se desarrollen.

- Cuando laboren trabajadores discapacitados en los centros de trabajo, las puertas, vías de acceso y de circulación, escaleras, lugares de servicio y puestos de trabajo, deben facilitar sus actividades y desplazamientos.

- Los edificios y elementos estructurales deben soportar las cargas fijas o móviles de acuerdo con la naturaleza de las actividades que en ellos se desarrollen, de tal manera que su resistencia evite posibles fallas estructurales y riegos de impacto, para lo cual deben considerarse las condiciones normales de operación y los eventos tanto naturales como incidentales que puedan afectarlos. 
- Techos. Deben:

a) Ser de materiales que protejan de las condiciones ambientales externas;

b) Utilizarse para soportar cargas fijas o móviles, solo si fueron diseñados o reconstruidos para estos fines; $y$

d) Soportar las condiciones normales de operación.

- Paredes. Deben:

a) Mantenerse con colores tales que eviten la reflexión de la luz, cuando se trate de las caras interiores, para no afectar la visión del trabajador.

- Pisos. Deben:

a) Mantenerse en condiciones tales que de acuerdo con el tipo de actividades que se desarrollen, no generen riesgos de trabajo;

b) Ser llanos en las zonas para el tránsito de las personas.

\section{- Escaleras. Deben cumplir con lo siguiente:}

a) Tener un ancho constante de al menos $56 \mathrm{~cm}$ en cada tramo recto $\mathrm{y}$, en ese caso, se debe señalizar que se prohíbe la circulación simultánea en contraflujo. Las señales deben cumplir con lo establecido en la NOM-026-STPS-1998;

b) Cuando tengan descansos, estos deberán tener al menos $56 \mathrm{~cm}$ para las de tramos rectos utilizados en un solo sentido de flujo a la vez y, de al menos, $90 \mathrm{~cm}$ para las de ancho superior;

c) Todas las huellas de las escaleras rectas deben tener el mismo ancho y todos los peraltes la misma altura, con una variación máxima de $\pm 0.5 \mathrm{~cm}$;

d) En las escaleras con cambios de dirección o en las denominadas de caracol, el peralte debe ser siempre de la misma altura;

e) Las huellas de los escalones en sus tramos rectos deben tener una longitud mínima de $25 \mathrm{~cm}$ (área de contacto) y el peralte una altura no mayor a $23 \mathrm{~cm}$. Las orillas de los escalones deben ser redondeadas (sección roma o nariz roma) y la distancia libre medida desde la huella de cualquier escalón, contemplando los niveles inferior y superior de la escalera y el techo, o cualquier superficie superior, debe ser mayor a $200 \mathrm{~cm}$.

f) Las huellas de los escalones deben contar con materiales antiderrapantes. 
- Escaleras con barandales con espacios abiertos. Las escaleras con barandales que cuenten con espacios abiertos por debajo de ellos, deben tener al menos una baranda dispuesta paralelamente a la inclinación de la escalera y cumplir con lo siguiente:

a) El pasamano debe estar a una altura de $90 \mathrm{~cm} \pm 10 \mathrm{~cm}$;

d) Los pasamanos deben ser continuos, lisos y pulidos;

e) En caso de contar con pasamanos sujetos a la pared, estos deben estar fijados por medio de anclas aseguradas en la parte inferior;

g) Cuando las escaleras tengan un ancho de 3 m o más, deben contar con un barandal intermedio y uno en los extremos;

h) Cuando las escaleras estén cubiertas con muros en sus dos costados, deben contar al menos con un pasamanos, y

i) Las edificaciones deben tener siempre escaleras o rampas peatonales que comuniquen entre nivel y nivel todos sus niveles, aun cuando existan elevadores o escaleras eléctricas.

- Rampas. La función de las rampas es de comunicar de un nivel a otro, con lo cual se tiene acceso a un punto elevado o bajo con objetos pesados o grandes con difícil acceso por escaleras y ayudan al tránsito de personas con alguna discapacidad motriz.Las rampas deben cumplir con las siguientes condiciones:

a) Las cargas que por ellas circulen no deben sobrepasar la resistencia para la que fueron destinadas;

b) No deben tener deformaciones que generen riesgos a los transeúntes o vehículos que por ellas circulen, sin importar si son fijas o móviles.

d) Deben tener el ancho suficiente para ascender y descender sin que se presenten obstrucciones en el tránsito de los trabajadores;

f) Cuando la altura entre el nivel superior e inferior exceda de $150 \mathrm{~cm}$, deben contar con barandal de protección lateral;

g) Cuando se encuentren cubiertas por muros en sus dos costados, deben tener al menos un pasamano. 
- Condiciones de seguridad en el funcionamiento de los sistemas de ventilación artificial. Debe cumplir con lo siguiente:

a) El aire que se extrae no debe contaminar otras áreas en donde se encuentren laborando otros trabajadores.

b) El sistema debe iniciar su operación antes de que ingresen los trabajadores al área correspondiente para permitir la purga de los contaminantes.

c) Contar con un programa anual de mantenimiento preventivo o correctivo, a fin de que el sistema esté en condiciones de uso.

- Ventilación de confort (No es de cumplimiento obligatorio, pero sí es necesario considerarlo). Para locales de los centros de trabajo, tales como oficinas, cuartos de control, centros de cómputo y laboratorios, entre otros, en los que se disponga de ventilación artificial para confort de los trabajadores o por requerimientos de la actividad en el centro de trabajo, se recomienda tomar en consideración la humedad relativa, la temperatura y la velocidad del aire, de preferencia en los términos siguientes:

- Humedad relativa entre el $20 \%$ y $60 \%$;

- Temperatura del aire de $22^{\circ} \mathrm{C} \pm 2^{\circ} \mathrm{C}$ para épocas de ambiente frío, y $24.5^{\circ} \mathrm{C} \pm$ 1.5으 para épocas calurosas; 3/4 Velocidad media del aire que no exceda de 0.15 $\mathrm{m} / \mathrm{s}$, en épocas de ambiente frío, y de $0.25 \mathrm{~m} / \mathrm{s}$ en épocas calurosas, y

- Se recomienda que la renovación del aire no sea inferior a 5 veces por hora.

Sería conveniente que en los programas de revisión y mantenimiento de los sistemas de ventilación se revisen parámetros como:

- La regulación del aire;

- El control de los caudales de ventilación;

- El aislamiento acústico;

- La limitación de la propagación de ruido;

- Las no condensaciones de la humedad, y

- La instalación eléctrica. 


\section{Norma Oficial Mexicana NOM-002-STPS-2010, condiciones de seguridad- prevención y protección contra incendios en los centros de trabajo (bibliotecas)}

El riesgo de incendio puede presentarse dentro de la biblioteca, ya que este inicia cuando se cuenta con materiales de rápida combustión (materia principal de la biblioteca) como papel, cintas, equipo de cómputo e instalaciones eléctricas, que si se encuentran en mal estado o sobrecargadas se torna un riesgo evidente, asimismo la falta de una revisión periódica, y el mantenimiento del orden y la limpieza incrementan el riego.

Existen diferentes tipos de fuego dependiendo el material que se quema. Los que aplican a la biblioteca son el de Tipo A, originado por material sólido, cuya combustión produce brasas e involucra materiales como papel, madera, basura, etc.; y el de Tipo $\mathrm{C}$, ocasionado por equipos energizados como instalaciones eléctricas, computadoras, subestaciones eléctricas, que producen cortos circuitos como motores, tableros, instalaciones eléctricas. Así pues el objetivo de esta norma es establecer los requerimientos para la prevención y protección contra incendios en los centros de trabajo.

\section{a. Obligaciones del patrón}

- Contar con un croquis, plano o mapa general del centro de trabajo, o por áreas que lo integran, actualizado y colocado en los principales lugares de entrada, tránsito, reunión o puntos comunes de estancia o servicios para los trabajadores, que contenga lo siguiente, según aplique:

1) La ubicación de los medios de detección de incendio, así como de los equipos y sistemas contra incendio;

2) Las rutas de evacuación, incluyendo, al menos, la ruta de salida y la descarga de salida, además de las salidas de emergencia, escaleras de emergencia y lugares seguros;

3) La ubicación del equipo de protección personal para los integrantes de las brigadas contra incendio, y

4) La ubicación de materiales y equipo para prestar los primeros auxilios. 


\section{b. Obligaciones de los trabajadores (bibliotecarios)}

- Cumplir con las medidas de prevención y protección contra incendios establecidas por el patrón.

- Participar en las actividades de capacitación y entrenamiento proporcionadas por el patrón para la prevención y protección contra incendios.

- Auxiliar en la respuesta a emergencias de incendio que se presenten en el centro de trabajo, conforme a la capacitación y entrenamiento recibidos.

- Participar en las brigadas contra incendio.

- Participar en los simulacros de emergencias de incendio.

\section{c. Condiciones de prevención y protección contra incendios}

- Vigilar que los extintores cumplan con las siguientes condiciones:

1) Que su ubicación esté en lugares visibles, de fácil acceso y libres de obstáculos;

2) Que cuenten con el sello o fleje de garantía sin alterar;

3) Que no existan daños físicos evidentes, tales como corrosión, escape de presión, obstrucción, golpes o deformaciones;

4) Que no existan daños físicos tales como roturas, desprendimientos, protuberancias o perforaciones, en mangueras, boquillas o palanca de accionamiento, que puedan propiciar su mal funcionamiento. El extintor deberá ser puesto fuera de servicio, cuando presente daño que afecte su operación, o dicho daño no pueda ser reparado, en cuyo caso deberá ser sustituido por otro de las mismas características y condiciones de operación.

- Contar con el registro de los resultados de la revisión mensual a los extintores que al menos contenga:

1) La fecha de la revisión;

2) El nombre o identificación del personal que realizó la revisión;

3) Los resultados de la revisión mensual a los extintores; y

4) Las anomalías identificadas. 
- Establecer y dar seguimiento a un programa anual de revisión y pruebas a los equipos contra incendio, a los medios de detección y, en su caso, a las alarmas de incendio y sistemas fijos contra incendio.

- Prohibir y evitar el bloqueo, daño, inutilización o uso inadecuado de los equipos y sistemas contra incendio, los equipos de protección personal para la respuesta a emergencias, así como los señalamientos de evacuación, prevención y de equipos y sistemas contra incendio, entre otros.

- Contar con rutas de evacuación que cumplan con las condiciones siguientes:

1) Que se encuentren libres de obstáculos que impidan la circulación de los trabajadores y demás ocupantes;

2) Que dispongan de dispositivos de iluminación de emergencia que permitan percibir el piso y cualquier modificación en su superficie, cuando se interrumpa la energía eléctrica o falte iluminación natural;

3) Que la distancia por recorrer desde el punto más alejado del interior de una edificación, hacia cualquier punto de la ruta de evacuación, no sea mayor de $40 \mathrm{~m}$. En caso contrario, el tiempo máximo de evacuación de los ocupantes a un lugar seguro deberá ser de tres minutos;

- Contar con salidas normales y/o de emergencia que cumplan con las condiciones siguientes:

1) Que comuniquen a un descanso, en caso de acceder a una escalera;

2) Que en las salidas de emergencia, las puertas abran en el sentido del flujo, salvo que sean automáticas y corredizas;

3) Que las puertas sean de materiales resistentes al fuego y capaces de impedir el paso del humo entre áreas de trabajo, en caso de quedar clasificados el área o centro de trabajo como de riesgo de incendio alto y se requiera impedir la propagación de un incendio hacia una ruta de evacuación o áreas contiguas por presencia de materiales inflamables o explosivos;

4) Que las puertas de emergencia cuenten con un mecanismo que permita abrirlas desde el interior, mediante una operación simple de empuje; 
5) Que las puertas consideradas como salidas de emergencia estén libres de obstáculos, candados, picaportes o cerraduras con seguros puestos durante las horas laborales, que impidan su utilización en casos de emergencia, y

6) Que cuando sus puertas sean consideradas como salidas de emergencia, y funcionen en forma automática, o mediante dispositivos eléctricos o electrónicos, permitan la apertura manual, si llegara a interrumpirse la energía eléctrica en situaciones de emergencia.

- Instalar extintores en las áreas del centro de trabajo, de acuerdo con lo siguiente:

1) Contar con extintores conforme a la clase de fuego que se pueda presentar.

2) Colocar al menos un extintor por cada 300 metros cuadrados de superficie o fracción, si el grado de riesgo es ordinario. Colocar al menos un extintor por cada 200 metros cuadrados de superficie o fracción, si el grado de riesgo es alto;

3) Colocarlos a una altura no mayor de $1.50 \mathrm{~m}$, medidos desde el nivel del piso hasta la parte más alta del extintor.

- Proporcionar mantenimiento a los extintores como resultado de las revisiones mensuales. Dicho mantenimiento deberá estar garantizado conforme a lo establecido en la NOM-154-SCFI-2005, o las que la sustituyan, y habrá de proporcionarse al menos una vez por año.

- Capacitación: Los trabajadores deberán ser capacitados para prevenir incendios en el centro de trabajo, de acuerdo con los riesgos de incendio que se pueden presentar en sus áreas o puestos de trabajo, en los aspectos básicos de riesgos de incendio y conceptos del fuego.

- Detectores de Incendio: Como regla general se recomienda instalar un detector por cada 80 metros cuadrados de techo, sin obstrucciones entre el contenido del área y el detector y una separación máxima de 9 m entre los centros de detectores (Tabla 1). 
Bibliotecas. Vol 35, № 1.1, enero-junio, 2017, pp.1-19. EISSN: 1659-3286

URL: http://www.revistas.una.ac.cr/index.php/bibliotecas/index

Tabla 1

Extintores contra incendio

\begin{tabular}{|l|l|l|}
\hline Agente extinguidor & Fuego Clase A & Fuego Clase C \\
\hline Agua & Sí & No \\
\hline Polvo químico seco, tipo ABC & Sí & Sí \\
\hline Polvo químico seco, tipo BC & No & Sí \\
\hline Bióxido de carbono (CO2) & No & Sí \\
\hline Halon & Sí & Sí \\
\hline Espuma mecánica & Sí & Sí \\
\hline
\end{tabular}

Fuente: Norma Oficial Mexicana NOM-002-STPS-2010, condiciones de seguridad-prevención y protección contra incendios en los centros de trabajo.

\section{NORMA Oficial Mexicana NOM-011-STPS-2001, Condiciones de seguridad e higiene en los centros de trabajo donde se genere ruido}

El ambiente de trabajo es esencial en el rendimiento humano, por lo que es necesario que el personal no trabaje más allá de los límites máximos de su resistencia y en condiciones ambientales adecuadas. Dentro de los factores físicos se encuentra el ruido. No todos los individuos tienen la misma resistencia al ruido, pero se dice que cualquier ruido superior a 90 decibelios perjudica.

El objetivo de esta norma es establecer las condiciones de seguridad e higiene en los centros de trabajo donde se genere ruido que por sus características, niveles y tiempo de acción, sea capaz de alterar la salud de los trabajadores; los niveles máximos y los tiempos máximos permisibles de exposición por jornada de trabajo, su correlación y la implementación de un programa de conservación de la audición.

\section{a. Obligaciones del patrón}

- Contar con el reconocimiento y evaluación de todas las áreas del centro de trabajo donde haya trabajadores y cuyo NSA sea igual o superior a $80 \mathrm{~dB}(\mathrm{~A})$, incluyendo sus 
características y componentes de frecuencia, conforme a lo establecido en los apéndices B y C.

Se establece los límites máximos permisibles de exposición de los trabajadores a ruido estable, inestable o impulsivo durante el ejercicio de sus labores, en una jornada laboral de 8 horas, según se enuncia en la Tabla 2.

TABLA 2

Límites máximos permisibles de exposición

\begin{tabular}{|c|c|}
\hline NER & TMPE \\
\hline $90 \mathrm{~dB}(\mathrm{~A})$ & 8 horas \\
\hline $93 \mathrm{~dB}(\mathrm{~A})$ & 4 horas \\
\hline $96 \mathrm{~dB}(\mathrm{~A})$ & 2 horas \\
\hline $99 \mathrm{~dB}(\mathrm{~A})$ & 1 hora \\
\hline $102 \mathrm{~dB}(\mathrm{~A})$ & 30 minutos \\
\hline $105 \mathrm{~dB}(\mathrm{~A})$ & 15 minutos \\
\hline
\end{tabular}

Fuente: NORMA Oficial Mexicana NOM-011-STPS-2001, Condiciones de seguridad e higiene en los centros de trabajo donde se genere ruido.

\section{NORMA Oficial Mexicana NOM-025-STPS-2008, Condiciones de iluminación en los centros de trabajo}

La importancia de tener en buenas condiciones los niveles de luz es porque tanto el exceso - la deficiencia pueden ser un factor para desencadenar accidentes y provocar enfermedades de trabajo. Cuanto mayor sea la concentración visual en detalles y minucias, más necesaria será la luminosidad. La unidad de medida de la iluminación es el lux, la medición a $75 \mathrm{~cm}$. del piso.

El objetivo de esta norma es establecer los requerimientos de iluminación en las áreas de los centros de trabajo, para que se cuente con la cantidad de iluminación requerida para cada actividad visual, a fin de proveer un ambiente seguro y saludable en la realización de las tareas que desarrollen los trabajadores. 


\section{a) Obligaciones del patrón}

- Contar con los niveles de iluminación en las áreas de trabajo o en las tareas visuales de acuerdo con la Tabla 2.

- Elaborar y ejecutar un programa de mantenimiento para las luminarias del centro de trabajo, incluyendo los sistemas de iluminación de emergencia.

- Instalar sistemas de iluminación eléctrica de emergencia, en aquellas áreas del centro de trabajo donde la interrupción de la fuente de luz artificial represente un riesgo en la tarea visual del puesto de trabajo, o en las áreas consideradas como ruta de evacuación que lo requieran.

- Niveles de lluminación para tareas visuales y áreas de trabajo. Los niveles mínimos de iluminación que deben incidir en el plano de trabajo, para cada tipo de tarea visual o área de trabajo.

\section{b. Evaluación de los niveles de iluminación}

- A partir de los registros del reconocimiento, se debe realizar la evaluación de los niveles de iluminación en las áreas o puestos de trabajo de acuerdo con lo establecido en el Apéndice A (NOM-025-STPS-2008).

- Determinar el factor de reflexión en el plano de trabajo y paredes que por su cercanía al trabajador afecten las condiciones de iluminación, según lo establecido en el Apéndice B, y compararlo con los niveles máximos permisibles del factor de reflexión (Tabla 3).

Tabla 3

Niveles de iluminación

\begin{tabular}{|c|c|c|}
\hline $\begin{array}{c}\text { Tarea Visual del Puesto de } \\
\text { Trabajo }\end{array}$ & Área de Trabajo & $\begin{array}{c}\text { Niveles Mínimos de } \\
\text { lluminación (luxes) }\end{array}$ \\
\hline $\begin{array}{c}\text { En interiores: distinguir el área } \\
\text { de tránsito, desplazarse } \\
\text { caminando, vigilancia, } \\
\text { movimiento de vehículos. }\end{array}$ & $\begin{array}{c}\text { Inferiores generales: } \\
\text { almacenes de poco } \\
\text { movimiento, pasillos, } \\
\text { escaleras, estacionamientos } \\
\text { cubiertos, labores en minas } \\
\text { subterráneas, iluminación de } \\
\text { emergencia. }\end{array}$ & 50 \\
\hline $\begin{array}{c}\text { Distinción moderada de detalles: } \\
\text { ensamble simple, trabajo medio } \\
\text { en banco y máquina, inspección }\end{array}$ & $\begin{array}{c}\text { Talleres: áreas de empaque y } \\
\text { ensamble, aulas y oficinas. }\end{array}$ & 300 \\
\hline
\end{tabular}




\begin{tabular}{|c|c|c|}
\hline $\begin{array}{c}\text { simple, empaque y trabajos de } \\
\text { oficina. }\end{array}$ & \\
\hline $\begin{array}{c}\text { Distinción clara de detalles: } \\
\text { maquinado y acabados } \\
\text { delicados, ensamble de } \\
\text { inspección moderadamente } \\
\text { difícil, captura y procesamiento } \\
\text { de información, manejo de } \\
\text { instrumentos y equipo } \\
\text { laboratorio. }\end{array}$ & $\begin{array}{c}\text { Talleres de precisión: salas } \\
\text { de cómputo, áreas de dibujo, } \\
\text { laboratorios. }\end{array}$ & 500 \\
\hline
\end{tabular}

Fuente: NORMA Oficial Mexicana NOM-025-STPS-2008, Condiciones de iluminación en los centros de trabajo.

Tabla 4

Niveles Máximos Permisibles del Factor de Reflexión

\begin{tabular}{|l|l|}
\hline \multicolumn{1}{|c|}{ Concepto } & Niveles Máximos Permisibles de Reflexión, Kf \\
\hline Paredes & $60 \%$ \\
\hline Plano de trabajo & $50 \%$ \\
\hline
\end{tabular}

Fuente: NORMA Oficial Mexicana NOM-025-STPS-2008, Condiciones de iluminación en los centros de trabajo.

c. Mantenimiento En el mantenimiento de las luminarias se deberá tomar en cuenta lo siguiente:

- La limpieza de las luminarias;

- La ventilación de las luminarias;

- El reemplazo de las luminarias cuando dejen de funcionar, o después de transcurrido el número predeterminado de horas de funcionamiento establecido por el fabricante;

- Los elementos que eviten el deslumbramiento directo y por reflexión, así como el efecto estroboscópico, y

- Los elementos de pre-encendido o de calentamiento. 


\section{Conclusiones}

La seguridad e higiene en México es un tema que no debe quedar fuera de los estudios de la bibliotecología, para así solicitar más apoyo a las autoridades de las que se depende para cumplir con la normatividad. Es necesario crear una cultura dentro de la comunidad bibliotecaria sobre la seguridad que conlleve al conocimiento y establecimiento de las normas del gobierno mexicano que evite riesgos al bibliotecario y usuarios de la biblioteca.

Todas las bibliotecas deben contar con una comisión de seguridad e higiene que cumpla con las verificaciones, registros, informaciones y revisiones en caso de reparaciones, adecuaciones o modificaciones que garanticen la seguridad del personal y usuarios. El personal como recurso vivo de la biblioteca está en la obligación de reportar anomalías en cuanto a seguridad a las instancias competentes en caso de ser detectadas, para evitar riesgos o accidentes posteriores.

Falta una normatividad propia a nivel nacional que trate específicamente sobre los edificios o espacios destinados a bibliotecas, por lo que se sugiere que las instancias competentes o las mismas universidades establezcan normas exclusivas para edificios o espacios de bibliotecas y que se actualicen conforme a las normas mexicanas. Se exhorta a los responsables y jefes de biblioteca a revisar la normatividad y hacer una evaluación respecto al cumplimiento de las normas para hacer los ajustes necesarios, ofreciendo la seguridad a todo el personal y usuarios de la biblioteca, con ello aminorando los riesgos.

\section{Bibliografía}

Arreola, R. (s.f.). Seguridad e Higiene industrial. Disponible en http://www.eumed.net/cursecon/ecolat/mx/2012/seguridad-higiene-industrial-mexico.html\# ftn1 Melnik, D. (2005). Bases para la administración de bibliotecas: organización y servicios. Buenos Aires: Alfagrama.

Reglamento Federal de Seguridad, Higiene y Medio Ambiente de Trabajo. Secretaría de Salud. Disponible en www.salud.gob.mx/unidades/cdi/nom/compi/r210197.html 
Rivera, M. (2004). La Aplicación de las normas de seguridad en las bibliotecas de la UNAM construídas en el periódo 1989-1997. México: UNAM.

Salgado, J. (2002). Higiene y seguridad industrial. México: Instituto Politécnico Nacional.

Secretaría del Trabajo y Previsión Social. NOM-001. Edificios, locales, instalaciones y áreas en los centros de trabajo. México.

Secretaría del Trabajo y Previsión Social. NOM-002. Condiciones de Seguridad-prevención y protección contra incendios en los centros de trabajo.México.

Secretaría del Trabajo y Previsión Social. NOM-011. (17 de abril de 2002). Condiciones de Seguridad e Higiene en los centros de trabajo donde se genere ruido.México.

Secretaría del Trabajo y Previsión Social. NOM-019. (13 de abril de 2011). Constitución, integración, organización y funcionamientos de las comisiones de seguridad e higiene.México

\section{Notas de los autores}

* Elsa Galicia Hernández: Mtria. en Administración en Organizaciones. Depto. de Planeación, Dirección General de Bibliotecas, UNAM, México elsag@dgb.unam.mx

** César S. Olguín Camacho: Mtria. en Bibliotecología y estudios de la Información. Coordinador de la Biblioteca del Programa Universitario de Estudios sobre la Ciudad, UNAM, México cesarenba@gmail.com

1 Esta normativa se encuentra disponible y descargable en formato electrónico desde http://www.stps.gob.mx/bp/secciones/dgsst/normatividad/n152.pdf

${ }^{2}$ Son los patrones o sus representantes, sindicatos titulares, trabajadores, comisiones de seguridad e higiene, supervisores de seguridad y médicos de las empresas y están obligados a cuidar la estricta observancia de este reglamento en sus respectivos centros de trabajo. 